De Tampere à Séville : bilan de la sécurité européenne $(2 / 2)$

\title{
Les parents immigrés pris « au piège » de la cité
}

Immigrant Parents Caught in the "Urban Trap"

\section{Olivier Masclet}

\section{(2) OpenEdition}

Journals

Édition électronique

URL : http://journals.openedition.org/conflits/812

DOI : $10.4000 /$ conflits.812

ISSN : $1777-5345$

Éditeur :

CCLS - Centre d'études sur les conflits lilberté et sécurité, L'Harmattan

Édition imprimée

Date de publication : 1 juin 2002

Pagination : 147-173

ISBN : 2-7475-3030-2

ISSN : 1157-996X

Référence électronique

Olivier Masclet, «Les parents immigrés pris « au piège » de la cité », Cultures \& Conflits [En ligne], 46 |

été 2002, mis en ligne le, consulté le 30 mars 2021. URL : http://journals.openedition.org/conflits/812 ; DOI : https://doi.org/10.4000/conflits.812

Ce document a été généré automatiquement le 30 mars 2021.

Creative Commons License 


\title{
Les parents immigrés pris « au piège » de la cité
}

\author{
Immigrant Parents Caught in the "Urban Trap"
}

Olivier Masclet

S'il est vrai que l'immigration transforme en profondeur les normes, valeurs et pratiques des familles immigrées, que les modèles éducatifs importés sont «travaillés par le temps passé en France ", peut-on pour autant en conclure que les "problèmes des jeunes» observables dans « les quartiers » (délinquance, toxicomanie, trafic, etc.) trouvent leur origine dans la «crise du modèle parental maghrébin » pour parler comme Michèle Tribalat ${ }^{1}$ ou dans « la faillite des pères maghrébins " pour reprendre l'expression de Pascal Duret ${ }^{2}$ ? Pour la première, «le décalage entre le modèle d'autorité traditionnelle et la loi française » expliquerait la «crise d'autorité » des familles maghrébines : privés de leur « toute puissance traditionnelle », les pères, occupant des positions professionnelles dévalorisées, se réfugient dans le silence, abdiquent leur " pouvoir » à leur épouse et à leurs enfants. Pour le second, "les grands frères" doivent leurs prérogatives à la "négation symbolique» de leurs pères déchus de leur statut, "humiliés sur leur lieu de travail », socialement disqualifiés et donc démunis du droit à la parole. Ces analyses illustrent assez bien l'espèce de doxaqui s'est peu à peu imposée dans les champs de la recherche et du travail social sur les familles immigrées. Les explications de type ethno-culturaliste s'imposent d'autant plus facilement qu'elles ont pour objet des populations qui ne disposent pas des ressources nécessaires pour répliquer aux discours tenus à leur propos. Attribuer la délinquance à « la crise d'autorité des familles maghrébines» et celle-ci au décalage entre les normes de la société française et des sociétés d'origine, n'est-ce pas considérer que ces familles sont en France « d'éternelles étrangères "? Dire que les pères maghrébins sont des pères "humiliés ", n'est-ce pas porter sur eux un regard réducteur, voire misérabiliste? Car un certain nombre d'entre eux ont fait leur "place » à l'usine, ont été syndiqués, ont contribué aux luttes contre les licenciements, la dureté des conditions de travail, pour l'amélioration des salaires, etc. Leur émigration a obéi à un espoir de survie - quitter le pays pour trouver du travail et fonder une famille - et ils l'ont conçue comme un moyen d'améliorer leur 
condition. D'ailleurs, leur projet de mobilité sociale force le respect de leurs enfants. Contre la vision des immigrés en termes de handicaps ethno-culturels, il faut aussi rappeler l'hétérogénéité des destins scolaires et sociaux des fils et filles d'une même famille. Toutes les familles maghrébines n'ont pas des enfants «livrés à eux-mêmes ", mais y compris au sein de celles où l'un des membres est devenu délinquant, les différences sont parfois très nettes entre les enfants ${ }^{3}$.

Cependant, pour rompre avec cette forme d'étiologie sociale qui impute la délinquance des « jeunes » aux propriétés culturelles des familles immigrées ou à « la dévalorisation de l'image du père ", il ne suffit pas d'opposer aux familles placées dans les situations les plus difficiles celles dont l'existence est moins problématique. D'une part, ce serait laisser dans l'ombre l'explication sociologique des difficultés bien réelles auxquelles nombre de familles immigrées vivant en cité sont aujourd'hui confrontées. D'autre part, ce serait occulter la démoralisation collective des parents immigrés - avec ou sans enfants devenus délinquants - relogés dans les grands ensembles. Réalisé à partir d'entretiens menés entre 1990 et 1995 avec des parents algériens et marocains habitant dans un quartier HLM de la banlieue parisienne ${ }^{4}$, cet article se propose de rendre compte des tensions que ces parents vivent, dont les causes ne sont pas «ethniques " mais sociales et économiques ${ }^{5}$. La réduction et la précarisation des emplois peu ou pas qualifiés, la mise en question de l'insertion professionnelle des jeunes à la sortie de l'école, la dévalorisation symbolique des emplois ouvriers, la discrimination à l'embauche et face au logement - qui détermine pour partie la "ghettoïsation » du quartier -, sont des facteurs de fragilisation. Les fils d'immigrés sans diplôme sont des «jeunes sans avenir $»^{6}$, qui n'ont d'autre affectation que le domicile familial ou la rue et la culture de rue $\mathrm{e}^{7}$.Ainsi peut-on comprendre l'angoisse de ces parents conduits à se protéger contre le quartier ${ }^{8}$. Comment préserver les jeunes désargentés des tentations de "l'argent facile»? Comment inciter les enfants à poursuivre des études quand tout autour de soi il est visible que la réussite scolaire n'évite pas le chômage ? Comment accepter le décalage souvent important entre les espoirs placés dans l'immigration et la réalité vécue? Questions douloureuses auxquelles ces parents répondent différemment en fonction de la situation objective de leurs fils et filles mais aussi de leur statut de père ou de mère.

Les effets sociaux et moraux des désordres

Les parents rencontrés sont rarement ceux dont les enfants ont «pris la mauvaise pente ». Ceux-là se taisent, évitent le sociologue - plus ou moins assimilé à un représentant des autorités - comme les situations susceptibles de réactiver la honte qu'ils éprouvent d'avoir un fils délinquant. Ceux qui parlent ont souvent de «bonnes raisons " de se plaindre, notamment d'être exposés au trafic, et condamnent des «jeunes» qui les menacent dans l'image qu'ils ont d'eux-mêmes. Logés dans les bâtiments les plus dégradés - faute d'un entretien suffisant - ils disent d'abord subir leur environnement. Les immeubles où ces parents vivent ne sont pas surveillés comme le sont les bâtiments gérés par la municipalité : les gardiens y sont moins nombreux, ils ne sont pas reliés à tout un dispositif de contrôle qui permet de limiter l'occupation des halls par les jeunes autant que le trafic. Des adolescents « squattent » leur hall, laissent les détritus de leur consommation nocturne (boites de coca et de bières, cartons de pizzas, mégots de cigarettes) et, de plus en plus, des dealers ont pris place dans leur cage d'escalier. A certains moments, la vie y devient un enfer : les toxicomanes laissent traîner les seringues, certains se « shootent» dans les recoins des escaliers, des tirs de pistolet se font entendre. Seule la mort par overdose ramène le trafic à des proportions 
moins visibles. Les familles n'osent pas ou plus intervenir pour défendre la tranquillité de leur cage d'escalier : elles ont peur des vengeances. Parce que le trafic se déroule tout près de leur domicile et qu'il est dirigé par des individus qui connaissent leurs enfants, de près ou de loin, elles sont tout particulièrement soumises à la «loi du silence ». Pendant notre enquête, la sœur d'un toxicomane mort par overdose a dénoncé des trafiquants à la police : quelques jours plus tard, sa voiture a été incendiée en signe de représailles.

Un sentiment de déshonneur

Pour les parents algériens et marocains relogés dans les années 1970, ce quartier représentait une "zone de deuxième implantation $»^{9}$ : territoire libéré de l'affiliation communautaire première, y accéder c'était emménager dans un "vrai» logement, quitter les cités de transit et les immeubles dégradés, avoir une vie à l'image de celle des ouvriers français. C'est aussi pourquoi ils acceptent de rencontrer le sociologue : conséquence de la stigmatisation, ils sont portés à témoigner qu'ils subissent eux aussi la dégradation et donc n'en sont pas responsables.

Ils perçoivent la dégradation du quartier comme un retour en arrière et cherchent désormais à le fuir : la concentration des familles immigrées dans les mêmes immeubles et l'accélération consécutive des déménagements des familles françaises annulant en quelque sorte les profits symboliques associés à l'inscription au sein d'un voisinage initialement mieux réputé. Le seul rassemblement des familles immigrées dans les mêmes secteurs de la cité les prive de leur capital d'ancienneté : "Avant, dans notre cage d'escalier, on était les seuls", disent-ils, pour signaler qu'ils sont arrivés parmi les premiers et qu'ils n'ont donc pas choisi d'habiter un « quartier immigré ». C'est malgré eux qu'ils ont vu s'installer les autres familles avec lesquelles ils disent entretenir peu de contacts. Les procédures d'attribution de logement font que les familles ne se connaissent pas vraiment, y compris celles originaires des mêmes pays: leur regroupement n'obéit pas à la solidarité familiale ou régionale qui unissait les familles dans les bidonvilles ${ }^{10}$ et, de fait, il est pour elles une assignation à "rester entre Arabes ». Ainsi les parents qui s'étaient battus pour quitter les bidonvilles éprouvent le sentiment d'un retour au "ghetto", mais à un "ghetto " où désormais domine le sentiment d'une promiscuité.

Cherchant à déménager, leurs demandes restent sans réponses. Ils savent plus ou moins consciemment que leurs chances d'accéder à un quartier mieux réputé et plus tranquille sont en réalité très faibles. Assignés aux secteurs les plus dévalorisés du marché du logement, certains manifestent alors une nostalgie à l'égard des cités de transit où ils avaient été relogés dans les années 1960-70 - autre ghetto qu'ils avaient voulu fuir. Ils disent que leur situation y était meilleure : outre que leurs espoirs étaient plus grands d'accéder à un "vrai» logement, on parlait moins de leurs enfants, les Arabes ne polarisaient pas autant l'attention, il existait des solidarités avec les Français qui ont peu à peu disparu. Il semble qu'ils se sentaient moins exposés au racisme : plus jeunes, récemment installés en France, entretenant le projet d'une amélioration de leur position résidentielle, voire professionnelle, et d'un "retour au pays ", ils pouvaient sans doute plus facilement refouler les blessures qu'il inflige. A présent, ils en ressentent toute la violence. Parce qu'ils habitent en France depuis de nombreuses années et qu'ils sont devenus les grands-parents de "petits français ", ils ressentent d'une manière particulièrement vive le rejet de leur groupe : «on nous met tous dans le même sac ». 
Plus anciennement installés que les autres immigrés du Maghreb, les Algériens manifestent tout particulièrement ce désarroi collectif. Les jeunes visibles, à l'origine des désordres, annulent la sympathie, voire la solidarité dont ils disent avoir bénéficié. Ils éprouvent le sentiment qu'on les confond aux « jeunes » qui se conduisent mal. Ils disent que leurs enfants n'ont pas d'honneur à défendre, qu'ils sont sans patrie, qu'ils se trompent en pensant être des Français. A leurs yeux, ils errent dans un no man's land identitaire qui les conduit à devenir des délinquants : refusant d'admettre qu'ils sont comme leurs pères, ils sont rejetés par les Français qui ne les voient pas à leur image. Nés dans les années 1930, les Algériens appartiennent à la génération qui a fait la guerre d'Algérie, sinon sur place, du moins en France tant ils ont subi la répression policière. Anciens colonisés qui ont conquis leur liberté, ils sont peut-être d'autant plus " atteints" par la délinquance des fils qu'elle les menace d'un retour collectif dans l'indignité. Quittant leur pays au moment où il se libère, ils ont voulu en être les ambassadeurs exemplaires : moyen pour eux de prouver leur appartenance à l'Algérie leur fidélité à la cause - autant que d'être reconnus en France comme des étrangers à part entière. Moins assurés que les autres immigrés dans leur identité nationale, ils sont à la fois plus exposés à la stigmatisation des origines et plus menacés dans leur honneur national.

Mais ce sont aussi les dépositaires d'une histoire nationale qui a mal tourné qui s'expriment dans les entretiens - celle de l'Algérie -, dont l'évolution les prive de ressources dans la construction d'une image de soi respectable. Ils sont de la génération des immigrés qui avaient «cru » en leur patrie et qui ont déchanté, qui, au bout de leur émigration, au terme de leur vie professionnelle, sont conduits à tirer un bilan d'autant plus amer de leur expérience que leurs enfants sont sans travail et que certains d'entre eux sont les « jeunes délinquants ». Défenseurs d'une cause nationale avortée, blessés dans leur identité d'ouvrier immigré respectable, ils peuvent alors être conduits à militer pour un civisme perdu : la religion étant à leurs yeux la solution pour ramener les jeunes à la raison et réhabiliter collectivement «les Arabes »; l'islam est pour eux un honneur de substitution.

Pères et mères face aux désordres

Plus au fait des situations vécues par les familles proches, les mères sont généralement moins portées que les pères à condamner publiquement les « jeunes qui traînent » ou les "parents qui les laissent tout faire». Elles font preuve d'une plus grande indulgence, ne serait-ce que parce que ces jeunes placés au centre de toutes les accusations pourraient être leurs fils. Parce que les mécanismes qui conduisent dans la délinquance ou dans la toxicomanie un enfant d'une fratrie sont obscurs et leur échappent, elles sont portées sinon à se sentir collectivement menacées, du moins à attribuer au hasard l'héritage d'un enfant difficile à élever. Incarnant le déshonneur des pères ouvriers immigrés, les jeunes délinquants bénéficient donc de leur compassion, sinon eux-mêmes, du moins celles qui les ont enfantés. Ce sont les mères qui maintiennent des relations avec les familles frappées par la délinquance des fils, opposant de fait à l'isolement provoqué par la honte une certaine solidarité.

Certaines mères que le sort a épargnées ne veulent pas quitter le quartier tant déménager serait pour elles un nouveau déracinement. Contrairement à leurs maris, qui sont de plus en plus des retraités (ou des préretraités) et qui " tournent en rond " dans leur domicile, elles sont "naturellement » à leur place à la maison et dans le quartier. Nombre d'entre elles sont à présent assistantes maternelles (elles reçoivent à 
leur domicile les enfants dont elles ont la garde) et c'est aussi leur emploi et leur statut de femmes salariées qu'elles défendent en défendant le quartier - les familles et les jeunes qui l'habitent. Contre leurs maris qui veulent déménager, qui envisagent de retourner au pays, plus ou moins définitivement, elles affirment vivre ici et refusent l'image négative de la cité entretenue par leurs conjoints sans activité.

Assistante maternelle depuis le départ en préretraite de son mari, Mme Brahim s'oppose ainsi frontalement à lui qui manifeste un dégoût pour les "jeunes casseurs » et n'hésite pas à accuser « les parents » de ne pas tenir leurs enfants. L'entretien avec ces parents s'est déroulé à l'issue d'une manifestation organisée par la municipalité en réaction au saccage du local du personnel chargé de la réhabilitation du quartier. $\mathrm{M}$ Brahim était l'un des rares habitants maghrébins présents : le poids de la honte interdit généralement aux Arabes de se manifester (et donc de manifester). Relativement isolé parmi la cinquantaine d'habitants français et d'employés municipaux qui serraient les rangs autour du maire et des adjoints, sa colère contre ces jeunes était visible. La discussion s'est facilement engagée entre nous: la dégradation du quartier et «les jeunes qui cassent tout » étaient les thèmes omniprésents. D'emblée, je suis frappé par sa sévérité à leur égard, mais aussi et peut être surtout, par l'extériorité avec laquelle il parle d'eux. Bien sûr, il ne connaît pas les « casseurs » qui ont agi le visage masqué (la rumeur dit néanmoins qu'ils sont arabes et de la cité), mais surtout il ne leur trouve " aucune excuse ", rien qui puisse lui permettre d'interpréter leur acte autrement que comme l'expression d'un pur plaisir à détruire. Il accepte presque sans réfléchir de me rencontrer pour discuter du quartier plus longuement, comme s'il voulait signifier toute la distance qui le sépare de ces jeunes, mais aussi son refus du fossé qui s'est creusé entre les Français qui se plaignent et les immigrés acculés au silence, qui se sentent culpabilisés.

Passé d'OS au rang d'ouvrier qualifié, ayant à son actif « 35 ans d'usine» (selon ses mots), M Brahim explique ne pas comprendre ces jeunes qui «ne travaillent pas » et qui semblent le menacer. Dès les premiers instants de l'entretien, il précise n'avoir jamais été au chômage et avoir donné « le goût du travail » à ses enfants. Ses propos résonnent de l'écho de l'indignation des Français, comme s'il lui fallait se donner en exemple pour bien montrer qu'on ne peut pas mettre «tous les Arabes dans le même sac ». Mais c'est aussi le quartier où sa famille s'est installée dès sa construction que ces jeunes saccagent, c'est-à-dire là encore le mérite associé au fait de figurer parmi les premiers locataires. C'est au fond tous les efforts fournis pour échapper à l'insécurité matérielle et s'établir que ces jeunes compromettent, abîment et dévaluent. Contrairement à son épouse, $\mathrm{M}$ Brahim ne peut leur manifester une quelconque solidarité : entre lui et eux, il y a toute la distance qui sépare l'ouvrier stable des sousprolétaires, les « ouvriers respectables » des « jeunes sans avenir ».

Pas plus que son mari, Mme Brahim n'approuve le saccage de l'antenne chargée de la réhabilitation, mais elle cherche néanmoins à comprendre les raisons pour lesquelles les jeunes l'ont commis. A ses yeux, ils ne sont pas des «voyous» mais des "jeunes privés de tout ». S'ils ne travaillent pas, ce n'est pas parce qu'ils refusent de le faire, mais c'est parce que le travail manque : rien ne leur est offert, pas même des petits boulots (entretenir la pelouse, balayer, etc.). Elle-même subit le bruit de ceux installés dans son hall, mais elle observe qu'ils sont sans autre affectation que la rue ou les abords des immeubles qu'ils tiennent à longueur de temps. Les repousser, c'est les exclure du seul territoire qu'ils possèdent. "S'ils cassent, c'est qu'ils se vengent », dit- 
elle, manifestant ainsi une solidarité à l'égard des jeunes démunis, exclus d'une vie normale.

L'idée que "les jeunes se vengent » est partagée par les mères qui constatent que leurs enfants " ne s'en sortent pas », y compris les mieux scolarisés d'entre eux qui, en dépit des études accomplies, restent sans emploi. «Il faut les comprendre » dit ainsi une autre mère algérienne de cinq enfants quelques jours après le saccage du local de la réhabilitation, qui explique "tout sacrifier " pour que ses enfants suivent des études (son mari est au chômage) et redouter qu'ils se découragent à cause du chômage et du racisme à l'embauche. Les mères entendent leurs enfants et les amis de leurs enfants dire qu'ils ne pas sont embauchés à cause de leur "gueule d'Arabe ", que "même les diplômés ne trouvent pas de travail », qu'habiter le quartier est une marque indélébile qui les exclut par avance, etc. Sans espoir, car privés de toute perspective professionnelle, les jeunes casseurs sont les porte-parole par défaut de leur protestation.

Soutenant les fils dont les pères ont honte, les mères préservent désormais jusqu'au bout leur progéniture ${ }^{11}$. Elles maintiennent des liens avec les fils incarcérés, ouvrent la porte de leur domicile malgré l'heure passée, protègent leurs garçons de l'hostilité du père. Mme Brahim est elle-même très liée à une autre famille algérienne de l'immeuble qui compte huit enfants dont deux qui ont «chuté » dans la toxicomanie : l'un est décédé du sida, l'autre cumule les condamnations. En prison, il suit une cure de désintoxication, mais de retour dans le quartier, il rechute. Excédé et malheureux, le père de ce jeune malade l'a chassé du domicile familial. C'est sa mère, aidée et encouragée par Mme Brahim, qui l'a « récupéré ».

La peur de la contamination par l'échec

Si les mères défendent leurs fils, elles ne peuvent cependant pas s'opposer à la dégradation des relations entre les familles et au sein même des familles, dégradation qui interdit toute forme d'intervention tendant à contrôler le voisinage et qui pousse à fuir la cité. Il y a eu une période heureuse (tant que les enfants étaient petits et facilement maîtrisables) à laquelle ont succédé «les temps difficiles »: vivre dans la cité, c'est aujourd'hui subir le racisme, les désordres engendrés par le développement du trafic de drogue et assister à la désorganisation des familles proches dont certains des enfants deviennent délinquants. Car si les familles désorganisées ont toujours existé dans le quartier, elles étaient jusqu'alors clairement identifiées et servaient de contre-modèle éducatif à la majorité des familles immigrées. Elles étaient les familles déviantes qui, par manque de ressources économiques et culturelles, "fournissaient " les délinquants. Il semble que l'extension et la durée du chômage aient profondément brouillé les cartes : la menace plane désormais sur toutes les familles qui ont encore des adolescents à domicile, sans travail, qui se découragent et à qui la cité peut procurer l'argent dont ils ont besoin ou la drogue pour « se défoncer " (s'oublier, fuir, rêver). Les familles se sentent vulnérables car tout autour d'elles les exemples se sont multipliés des jeunes des « familles respectables » qui se laissent entraîner.

Des jeunes « démotivés»

Installée dans la cité depuis 1971, Mme Afif appartient à ces familles algériennes dont aucun enfant n'a " mal tourné ». Mais de fait elle ne peut pas se sentir complètement protégée des risques inhérents à la situation actuelle des jeunes sur le marché du travail et tout particulièrement des jeunes fils d'immigrés maghrébins non-diplômés ou détenteurs de titres scolaires dévalués, victimes dans tous les cas de la discrimination 
«au faciès" ou à cause d'un nom "pas comme il faut». Elle côtoie tous les jours des mères qui n'ont pas eu sa chance et dont les enfants sont toxicomanes ou délinquants : "la France fait des enfants des drogués", dit-elle, pour dire sa peur face au risque de déchéance.

Cette mère s'était «blindée » contre la cité. Elle explique avoir imposé à ses enfants de ne pas rester dans la rue après l'école : inscrits à un club de sport (le judo), ils étaient tenus les mercredis. Aussitôt rentrés de l'école, elle les mettait en pyjama : moyen pour elle de réduire au maximum le temps concédé aux autres enfants. Elle voulait qu'ils réussissent. Bien qu'elle-même n'ait pas été scolarisée, elle suivait néanmoins de très près leurs devoirs. Elle faisait partie de ces mères immigrées présentes à toutes les réunions avec les enseignants qui n'hésitaient pas à prendre rendez-vous avec eux pour "faire le bilan ». Mme Afif explique qu'elle ne donnait jamais tort aux enseignants et imposait à ses enfants qu'ils se plient à la discipline exigée. Mobilisée autour de l'école, cette famille n'a pas fait construire de maison au pays : le budget familial a été consacré à préparer l'avenir des enfants. Très vite donc, elle a aussi abandonné les voyages annuels en Algérie. Outre que les enfants manifestaient de plus en plus leur distance, les parents ont choisi de ne plus épargner comme ils le faisaient pour les vacances mais d'« offrir des études " à leurs enfants. Ses six enfants vivent à son domicile (le dernier est âgé de deux ans). Trois d'entre eux ont poursuivi des études supérieures, conformément aux attentes de leurs parents. L'un des cadets prépare un bac professionnel. Mais un autre a interrompu sa scolarité avant son BEP. La cité angoisse donc d'autant plus que Mme Afif a le sentiment de ne plus pouvoir s'en protéger : les aînés ont mieux réussi à l'école que leurs petits frères, comme si les scolarités dépendaient elles-mêmes de plus en plus de la cité.

Les aînés de cette famille mettaient sans doute d'autant plus d'ardeur à travailler à l'école que leurs parents attendaient plus fortement d'eux qu'ils se conforment à leurs attentes. Il se pourrait que dans les familles ouvrières nombreuses, les derniers-nés soient moins sous la pression parentale, le flot des injonctions. De plus, en concurrence avec leurs aînés qui ont suivi la voie indiquée, ils supportent peut-être plus difficilement les effets négatifs des sanctions scolaires qui s'exercent au sein même de la famille. Parce que toute famille est un champ de relations, les cadets jugés moins bons que les aînés sont disposés à investir d'autres jeux. Le cadet de cette famille qui n'a pas réussi à l'école veut se lancer dans la mode : univers professionnel où il pense que les diplômes sont moins nécessaires. Dans l'attente de le faire, il est sans emploi du temps.

Mais au-delà de son cas, il semble que les adolescents nés après les années 1975 soient effectivement moins portés à "s'accrocher à l'école ». Voyant sans doute que "même les diplômés ne s'en sortent pas ", ils ne sont pas incités à le faire. L'illusion qui avait pris corps autour de la valeur du diplôme pendant les années 1980 s'est dissipée ${ }^{12}:$ si les enfants d'immigrés de la cité nés entre 1965 et 1975 - premiers «bénéficiaires » de l'allongement des scolarités - croyaient possible d'accéder à des titres scolaires " porteurs » sur le marché du travail, leurs successeurs ont intériorisé que les diplômes préparés sont dévalués, qu'ils n'ont pas la valeur escomptée. Ils sont donc «naturellement » moins enclins à se conformer à l'ordre de l'Ecole. Les parents disent qu'ils ont "plus de mal avec les petits qu'avec les plus grands». Ils sont plus indisciplinés : ne croyant plus assez que "l'Ecole sert à réussir ", ils ne peuvent pas accepter les recommandations parentales aussi bien que les règles élémentaires de l'institution scolaire. Les enseignants du collège disent aussi qu'ils sont plus chahuteurs 
et notent la différence avec les plus grands, qui se tenaient mieux en classe, manifestaient moins d'insolence, discutaient moins les notes. Ils disent que la ruse pour réussir s'est peu à peu généralisée. Parce que le baccalauréat est à la fois un titre dévalué et le passeport nécessaire à l'accès au marché du travail («sans le bac, t'es mort " disent les adolescents qui ont intériorisé les normes d'accès au marché du travail, y compris aux emplois non qualifiés), les élèves sont à la fois peu portés à le reconnaître et déterminés à l'obtenir. Ils ne peuvent plus supporter les mauvaises notes, les «sales jugements" des enseignants ou les orientations vers les voies de garage. Le collège est un terrain miné de relations entre adolescents et enseignants, où les uns tentent d'" arracher " aux autres les "bons points " qu'ils sont conduits à leur refuser dans le seul objectif d'éviter le LEP et d'aller jusqu'au bac. Moins disposés que leurs aînés - qui croyaient en l'égalité des chances - à reconnaître la légitimité des sanctions scolaires, les cadets exercent une pression sans relâche sur les professeurs. Ainsi les « mauvais élèves » bénéficient-ils d'une complicité et d'une solidarité d'autant plus grande de la part des autres adolescents qu'ils appartiennent à une même génération. Génération qui à la fois ne croit plus dans l'Ecole et n'a pas d'autre solution que d'y rester. Les parents tentent désespérément de soustraire leurs enfants à l'influence des autres adolescents, seule solution pour les « re-motiver ».

Des parents menacés par la galère de leurs fils

On peut alors comprendre pourquoi certains entretiens avec les parents immigrés revêtent la forme d'un appel au secours, appel de parents qui tentent de fuir la cité pour préserver leurs enfants de la délinquance et qui attendent implicitement du sociologue qu'il intercède en leur faveur auprès de la mairie et des services sociaux. C'est le cas des parents Iza, qui ont six enfants dont deux, âgés de 20 et 22 ans, sortis de l'école sans qualification. S'ils ont accepté de parler de leurs relations avec leurs fils sans emploi, c'est en effet parce qu'ils cherchent à les extraire du "bain de la cité ", où ils s'enfoncent peu à peu. L'un d'eux, accusé de viol en réunion, a été emprisonné pendant six mois. Le jugement l'a disculpé, mais pour ses parents, il ne fait aucun doute qu'il est menacé.

Aucun de leurs six enfants n'a poursuivi d'études supérieures. L'âné (né en 1970) est diplômé d'un CAP électrotechnique. Les deux filles nées ensuite (1972 et 1975) sont vendeuses : l'une au Mc Donald's, l'autre dans un magasin de vêtements de la Défense. Les deux garçons suivants (nés en 1976 et 1977) ont arrêté l'école après avoir échoué au CAP. Le dernier enfant (né en 1979) est apprenti cuisinier. L'échec scolaire des enfants de cette famille est peut-être pour partie l'un des effets des conditions de l'installation en France des parents et plus spécialement de M Iza. Fils de paysans pauvres, il est « envoyé » en France par son père qui ne peut plus subvenir à ses besoins. Il garde un souvenir assez malheureux des trois années passées à l'école à son arrivée : relégué dans une classe qui regroupait des enfants plus jeunes que lui, il s'y est senti profondément mal à l'aise et a d'emblée été en échec. Son expérience malheureuse de l'école le prédisposait sans doute moins que les parents immigrés de la première génération à tout en attendre. A 16 ans, M Iza est embauché aux usines Wonder où il est sous la coupe de contremaîtres autoritaires. Il occupe différentes places dans différentes usines avant de se stabiliser dans une entreprise de câblage où il passe d'OS à chef d'équipe. Il y reste 22 ans. A la suite d'un licenciement économique, il devient chauffeur livreur. Quant à Mme Iza, aînée d'une famille de huit enfants, elle n'a pas pu se soustraire à l'ordre domestique et aux attentes traditionnelles à l'égard des filles de 
sa génération: elle stoppe sa scolarité avant le CAP. Certains de ses frères, nés et scolarisés en France, ont poursuivi des études supérieures.

Si les enfants de cette famille n'ont pas de diplômes professionnels, les filles semblent moins handicapées que leurs frères pour accéder au marché du travail : toutes les deux occupent un emploi de vendeuse. Sur le marché des services - secteur désormais dominant du marché du travail ouvert aux jeunes des classes populaires - elles peuvent plus facilement négocier leur capital corporel. Placées en contact avec les clients, les femmes sont plus recherchées que les hommes pour occuper les emplois de ce type qui valorisent les ressources relationnelles de "la féminité » (i.e.historiquement et socialement construite). Leurs frères peuvent moins y prétendre et sans doute d'autant moins qu'ils ont «une gueule d'arabe». Ce sont les hommes qui concentrent la stigmatisation des « Arabes » : outre qu'ils ne peuvent maquiller leur apparence comme le font les femmes, ils représentent la menace du danger des cités ("incivilités", langage ordurier, risque de "violence", etc.). La discrimination à l'embauche (indissociablement sexuelle, sociale et fondée sur l'origine ethnique) explique pour partie que les filles manifestent plus de «courage professionnel » que les garçons découragés à l'avance. Mais leur « volontariat professionnel » est aussi le produit des dispositions acquises dans la famille. Bien plus que leurs frères, les filles ont appris à servir, à se plier aux injonctions parentales les appelant à "se rendre utiles »: dispositions domestiques transférables et valorisées sur le marché des services. Moins reconnus «capables» que leurs sœurs d'occuper les emplois de service, les garçons sans diplôme sont également moins portés qu'elles à le faire.

Les fils démunis de capital scolaire n'ont que leurs bras pour ressources. Or non seulement les emplois qui les réclament (manœuvres, manutentionnaires, caristes, etc.) se sont raréfiés, mais de plus les emplois existants sont aujourd'hui occupés par des jeunes qui ont poursuivi une scolarité. Les jeunes démunis scolairement subissent directement la concurrence des jeunes éliminés moins précocement de la compétition scolaire, jeunes plus «employables» aux yeux des patrons, "mieux adaptés » aux normes professionnelles : plus ponctuels au travail, ils contestent moins l'autorité de leurs supérieurs ou ne le font pas sur le ton menaçant des jeunes qui n'ont pas d'autre ressource opposable que leur virilité ${ }^{13}$. Il semble donc, que le chômage s'étendant, les employeurs aient finalement eu raison des "jeunes rétifs » à l'ordre du travail : ils peuvent puiser dans le vaste réservoir des jeunes « aux normes », la main d'œuvre dont ils ont besoin.

Les parents Iza constatent que leurs fils ne "cherchent pas à travailler » : seul «le vieux part au boulot », les fils « restent au lit », disent-ils. Semblant anticiper un refus ayant intériorisé leur absence de valeur professionnelle - ils ne rentrent pas dans la course au travail : ils ne sont pas inscrits à la mission locale, ne prennent pas la peine de lire les petites annonces, ne font aucune démarche auprès des entreprises, etc. Passivité que leur père ne peut pas admettre : outre qu'ils dépendent de lui pour se nourrir, à leur âge il travaillait depuis plusieurs années et avait accepté «tous les boulots »- manifestant un courage dont à ses yeux ses fils sont dépourvus. Son ton dit assez qu'il les juge fainéants, voire totalement immatures. M Iza appartient à la génération des ouvriers pour qui devenir ouvrier s'imposait à la manière d'une évidence : destin des enfants éliminés précocement de l'école, mais destin accepté. La généralisation du passage dans le secondaire, l'allongement des scolarités, la massification des bacheliers ont depuis métamorphosé le rapport à «la condition ouvrière $»^{14}$. Si ses fils ne cherchent rien - et en tout cas pas un « boulot d'ouvrier » - ce 
n'est donc pas seulement parce que leurs chances sur le marché du travail sont très faibles, mais c'est aussi parce que l'accès à la classe ouvrière s'effectue désormais par l'Ecole : «On devient ouvrier parce que l'on est 'mauvais' à l'école, parce que l'on est bête ». En refusant d'occuper les emplois auparavant acceptés par les fils d'ouvriers, les fils de M Iza manifestent un refus de la dévalorisation sociale, qui est aussi une dévalorisation de l'image de soi $^{15}$.

Parce que devenir ouvrier revêt pour les fils démunis scolairement un sens qui n'est plus que négatif, ils prolongent l'indétermination de leur statut: la jeunesse est pour eux un refuge. Les fils Iza sont deux jeunes de 20 ans qui entretiennent leur «look». Les filles de leur groupe diraient qu'ils sont " mignons ", ils soignent leurs cheveux que l'un d'entre eux porte longs (s'identifiant plus aux jeunes des couches moyennes qu'aux "gars de la cité » qui se font presque raser le crâne) et font attention au choix de leurs habits. "La sape " (habits de marque) les inscrit dans la culture jeune, les rapproche symboliquement des adolescents plus fortunés et ainsi cache la réalité de leur condition. Jeunes mais pauvres (financièrement et culturellement), ils sont voués à la culture des rues tout en ayant intériorisé les normes dominantes de consommation et de manières d'être juvéniles. Le fils de cette famille impliqué dans un viol collectif est celui qui manifeste des troubles du langage et qui a le plus rapidement décroché de l'école. Il se pourrait qu'il se soit d'autant plus facilement laissé entraîner qu'il est plus particulièrement démuni et exposé aux jugements négatifs.

Que faire des fils sans emploi?

Si mettre à la porte du domicile familial les jeunes «rétifs au travail» a longtemps représenté la seule solution qui s'offrait aux pères ouvriers pour contraindre leurs fils à se prendre en charge, $M$ Iza ne peut pas s'y résoudre: ce serait condamner définitivement ses fils à la rue et à la déchéance ${ }^{16}$. Le chômage de masse prive les pères d'un argument « de poids ». Indépendamment des dispositions de leurs fils à l'égard du travail ouvrier, ils peuvent d'autant moins les rappeler à un principe de réalité que les emplois sont rares. Ces parents sont contraints de les garder à la maison, de subvenir à leurs besoins, de poursuivre sans fin visible tout le travail domestique sans lequel leurs fils seraient tout à fait livrés à eux-mêmes. Ils les protègent autant qu'ils peuvent parce qu'à leurs yeux, ils ne sont pas complètement coupables de ne pas en chercher. Comme les autres parents, ils voient que "même les diplômés ne s'en sortent pas ». Comment pourraient-ils reprocher à leurs fils d'être au chômage? Mais s'ils manifestent publiquement qu'ils les soutiennent, c'est peut-être aussi parce qu'ils s'efforcent de se convaincre qu'ils n'ont vraiment pas d'autre choix que celui de les garder à la maison. L'aide qu'ils leur apportent ne va pas de soi, elle les oblige en permanence à se contrôler pour ne pas les «braquer». Redoutant que leurs fils leur échappent complètement, ces parents ne peuvent que réduire les tensions avec eux et accepter une vie familiale dégradée.

Garder à domicile des adolescents de 20 ans déscolarisés et sans emploi, c'est en premier lieu devoir accepter leur présence fantôme. Leurs fils sont «là ", ils rentrent aux heures imposées par M Iza, mais en même temps ils sont absents, étrangers à « la vie de famille ». Privés d'un emploi du temps régulier, ils se lèvent tard et ne prennent plus les repas avec leurs parents. Ils s'isolent, ignorent leurs «vieux», vaquent aux seules occupations qui les retiennent à la maison : ils écoutent de la musique, regardent la télé, utilisent l'appartement plus qu'ils l'habitent. Ils évitent surtout de croiser les regards de leurs parents : la froideur des relations familiales résulte des évitements 
multiples qui sont « le prix à payer » pour maintenir des rapports pacifiés entre parents et jeunes "figés " à un stade adolescent. Les parents Iza disent qu'élever le ton reviendrait à durcir les relations. Ils évitent donc de laisser (trop) transparaître leur désarroi, voire leur colère en les voyant " traîner toute la journée ». Ils observent leur ennui sans pouvoir les mobiliser: toute intervention de leur part risque d'être reçue par leurs fils comme des « leçons de morale » et peut donc contribuer à les éloigner un peu plus de « la maison», dernier abri avant la rue.

Privés d'autonomie financière, leurs fils dépendent des soutiens familiaux : leur mère, leur sœur, leur frère leur fournissent l'argent de poche dont ils ont besoin. Comme elle le faisait quand ils étaient enfants, leur mère les habille. Ce sont les parents qui "courent à leur place " pour les habiller et les nourrir, dit-elle, s'ingéniant à leur trouver « les marques qu'il faut » pour les protéger des «tentations de l'argent facile ». Elle semble également les protéger contre leur père ou, plus précisément, prévenir les conflits entre eux et lui. En disant à son mari que ses fils le respectent et n'abusent pas de lui, elle tente de préserver son estime de père, chef d'une famille qu'il ne contrôle plus. Mais ces parents qui veulent sauver leurs fils de « la galère » semblent se préparer " au pire " tant la cité offre des sources de revenus clandestins face auxquels ils sont impuissants. Le trafic et le manque d'emplois accessibles redoublent l'effet propre à la condition des dominés : ces parents sont conduits à se résigner, voire au fatalisme ${ }^{17}$.

Le retrait des pères algériens

Si l'arrestation des fils - coupables de trafic, de vandalisme, de vol, d'usage de stupéfiants - plonge les parents dans la honte, elle précipite aussi le retrait des pères et peut-être plus spécialement des pères algériens. Retrait symbolique de pères qui cohabitent avec des «mauvais fils » qu'ils ne regardent plus, retrait physique de ceux qui retournent «sur leurs terres ». Mais l'arrestation des « fils indignes » n'est jamais l'acte décisif, ultime révélateur de la distance qui sépare les parents de leurs enfants nés et socialisés en France, elle est plutôt le signe insupportable du fossé qui s'est creusé entre eux. Les fils délinquants sont les fils doublement illégitimes des pères algériens qui redoublent en intensité les sentiments contradictoires qu'ils entretiennent à l'égard d'eux-mêmes (de leur émigration) et de leurs enfants en lesquels, écrit Sayad, «ils ne se reconnaissent pas et que, par conséquent, ils ne peuvent reconnaître pleinement $»^{18}$.

Le père de NaïmaA l'occasion de son dernier voyage en Algérie, le père de Naïma a dit à sa famille qu'il ne comptait plus revenir, qu'il ne serait pas là "pour les anniversaires" (moment où traditionnellement la famille se rassemblait). Il n'a pas supporté de voir son fils escorté par les policiers : accusé de vandalisme, il lui a fait honte et " il l'aurait tué ", dit Naïma, si sa mère ne l'avait pas protégé. Son père " en a eu marre de lui " et c'est pour ça qu'il est parti, explique-t-elle. Mais ce « mauvais coup » est intervenu après une longue série de « rêves » déçus, rêves de promotion qui n'ont pas été accomplis, faits d'un désir intense de revanche, dont l'échec le fait souffrir et plonge ses enfants, qui n'ont pu ni satisfaire ni répudier les attentes de leur père, dans le désarroi.

Contrairement à d'autres familles immigrées maghrébines, qui accordaient moins d'importance à la scolarité des filles, le père de Naïma veut que ses quatre enfants réussissent, indépendamment de leur sexe ${ }^{19}$. Aînée, Naïma est de fait plus particulièrement sous l'emprise des attentes paternelles : il la voit médecin, profession noble qui consacrerait la réussite de toute la famille. Au moment de l'entretien, Naïma 
travaille à l'usine. Ouvrière intérimaire, elle est sur chaîne. Elle a échoué deux fois au baccalauréat et occupe cet emploi dans l'attente d'une formation qui la conduira à un poste plus qualifié. Elle a honte d'être une simple ouvrière d'usine. A ses yeux, son «niveau terminale » aurait dû la protéger d'un emploi de ce type et elle a conscience de ne pas être à la hauteur des ambitions de son père et de son statut dans la famille. "Privilège-piège ${ }^{20}$, sa position d'aînée, dont elle n'a pas pu satisfaire les attentes, la contraint à mentir sur sa situation réelle à ses parents et à ses frères, " prix à payer " pour préserver son estime de soi.

«Mon père il nous a largués, enfin, il a décidé là, de rester plus longtemps en Algérie. Ça fait déjà cinq mois qu'il est là-bas et quand on lui téléphone, il dit qu'il est mieux là-bas. En septembre, quand ma mère est revenue, elle m'a dit qu'il restait là-bas pour des travaux. J'y ai cru sur le coup. Puis un mois après, il était toujours pas là pour les anniversaires. Chez nous, septembre et octobre, c'est les anniversaires, ma sœur, mon père, mon frère et moi. Je croyais qu'il allait revenir. En fin de compte, le premier anniversaire, celui de Ouardia, il l'a souhaité au téléphone. On lui a posé la question: ' Tu viens pas?', ' Non j'suis bien là-bas, j'reste pour le moment '. ' Pour le moment, ça veut dire quoi ?', ' Oh ! J'sais pas, quand j'aurai envie de revenir je reviendrai '. Ça veut tout dire. Après on l'a appelé, nous, pour son anniversaire : 'Tu viens ?', 'Non, non, j'ai pas envie de venir, vous êtes assez grands pour vous démerder'. Et voilà ! Ma mère, elle s'en fout, elle a toujours vécu dans la misère, alors elle est débrouillarde: elle bosse toute la journée, elle rentre, et puis voilà. Elle essaye d'oublier.

Ma mère, c'est une bosseuse, toute sa vie elle travaillera. Elle s'est démerdée pour apprendre le français, dès qu'elle est arrivée elle a bossé, elle a passé son permis, elle l'a eu. Elle, c'est toujours apprendre, apprendre, jamais de recul, toujours en train d'apprendre, toujours à courir à droite à gauche après l'argent. Parce que, bon, mes parents ont investi là bas pour une maison, donc il fallait trouver de l'argent. Papa, vu les diplômes qu'il a, il était manutentionnaire, 35 ans dans la même boite, t'imagines? Après il était préretraité et il est resté à la maison pendant deux ans ici. J'vais te dire, c'était pas drôle. Il est finalement parti dans sa super baraque cotée à 200 millions, deux étages, terrasse, dix pièces, $300 \mathrm{~m} 2$... Ils espéraient qu'on irait (rires)! Mon frère Hocine, ça fait au moins quatre ou cinq ans qu'il n'a pas mis les pieds là-bas. Nous, on y est allé cet été. Bon, on y va parce qu'il y a les grands-parents, j'ai mes grands-mères, mes tantes... C'est surtout par respect pour eux et pour mes parents, pour pas qu'ils disent qu'on renie... C'est pas qu'on renie, mais eux, ils l'interprètent de la sorte. C'est même pas un refus, parce qu'on accepte les gens, leur manière de vivre. Mais on ne veut pas vivre comme eux, donc... On est différents quoi, c'est tout, et ça, ça passe pas. Alors, au départ mes parents réagissaient mal. C'était toujours: ' Ne vous considérez pas comme Français '. ' Tôt ou tard, ils vous redemanderont vos cartes d'identité '. ' Ça se voit sur vos visages que vous n'êtes que des Maghrébins '. Nous, on leur dit que ça veut rien dire, qu'il y a les compétences derrière. Mon père c'est: ' T'inquiète pas! Quand ils en auront marre, ils vous jetteront et vous reviendrez de l'autre côté '. Nous, aux parents, on leur répondait : 'Vous dîtes n'importe quoi, on est bien acceptés, on n'a pas de problèmes, les gens qui parlent ce sont ceux qui ont des problèmes. Quand tu cherches du boulot, t'es pas rejeté. A l'école, on te rejette pas parce que t'es beur, c'est pas automatique. Mais mon père est très politique. Tu le vois comme ça, tu crois qu'il est Français, il aime bien la politique, il aime parler. Mais bon, paraît que quand tu vieillis tu retrouves tes racines (...). Mais tu vois, il pouvait parler comme ça à certains moments et en même temps, c'était l'école, l'école, l'école. L'école et le sport. Ecolesport. Comme ça, t'es occupé $24 \mathrm{~h}$ sur 24 (rires). Il avait des rêves... Mon père, il nous 
voyait avec des supers métiers, mais immenses quoi, trop, beaucoup trop... Moi, il voulait que je sois médecin. Hocine, il le voyait architecte, Ouardia, avocat. Et Kamel, il le voyait mécanicien. Mon petit frère a très vite eu des problèmes à l'école. Il a été scolarisé pendant trois ans dans une école orthophonique. Alors mon père n'avait pas pour lui des projets trop grands. Mais quand même, il disait que la mécanique c'est ' toujours utile ', qu'un ' bon mécanicien ça gagne bien sa vie '... C'est sûr qu'on l'a déçu. Ils ne savent pas là que je bosse en intériM Ma mère le sait, mais elle ne sait pas quel boulot je fais. Ce serait trop les décevoir. Il pensait me voir grande: ' médecine ', ' médecin'. Il voyait grand pour moi. Donc... C'est tout à côté quoi. Bon, j'travaille là, c'est histoire de gagner un peu d'argent et puis c'est tout. Mais bon, il l'aurait mal vu, mal interprété. Je préfère qu'il ne dise rien. Je dis que je bosse et puis c'est tout. Ma sœur sait où je bosse, mais mon frère Hocine, je ne lui dis pas. Je préfère pas qu'il sache. Même à l'autre, j'ai rien dit. Ils savent que je suis en intérim et c'est tout. C'est plutôt par fierté, par orgueil, je sais pas. J'ai toujours été la référence, donc si je leur dis que je travaille là dedans, ils vont dire : ' Putain, elle est descendue bas '. C'est vrai. Bosser à la chaîne, bon, normalement, c'est que les gens sans qualification qui y bossent (...).

Mon père nous aurait vus mieux quoi. Alors il est déçu, il nous demande pas ce qu'on fait parce qu'il ne veut pas voir que ses rêves ont raté. Bon, Hocine a quand même réussi un BTS par alternance. Là, il travaille au Club Méditerranée, il est pas souvent avec nous. Ouardia, elle a eu son bac cette année, elle est prise à l'IUT. C'est pas tout raté quand même. Moi, je vais faire un BTS par alternance bientôt, je ne vais pas rester comme ça. Y'a que mon frère Kamel qui n'a rien, qui n'est pas allé jusqu'en terminale. Et là c'est plus grave, parce que lui, il n'a vraiment rien du tout, j'vois pas trop ce qu'il peut faire. (...) Il a quoi? Un niveau BEPC. Moi, je voulais pas le pousser au niveau du lycée, je voulais qu'il soit clair dans ce qu'il voulait. Alors, au départ c'était électronique. Comme son dossier n'était pas correct, il a été refusé. Son niveau de mathématiques était trop faible pour rentrer en BEP. Donc il a demandé autre chose. Autre chose c'était quoi ? La mécanique. Pareil. Fallait être bon en physique, donc il a été refusé. Donc ils ont pas cherché à comprendre, ils l'ont foutu dans le BEP qu'il y avait, c'était CAS, du secrétariat et de la compta. Il a tenu une année et à la fin de la première année, il a dit : ' c'est pas mon truc'. On lui disait : non, continue, décroche au moins ton BEP, après on verra. ' Non, on ne verra pas, c'est pas mon truc, pourquoi je vais faire un BEP qui ne me plait pas, de toutes les manières, je ne l'aurai jamais '. Et il ne s'est pas trompé... Bon, c'est grave, il fait rien, il multiplie les conneries et... Mon père aussi, il réagit mal. Avant l'été, les flics sont quand même venus à la maison parce qu'il a été accusé d'avoir mis le feu à la Vieille ferme (vieux marché couvert du quartier ancien de la ville). Soit disant qu'il n'y est pour rien, que des types auraient fait ça parce que la mairie n'a pas voulu donner d'argent à leur association. Mais bon, mes parents ont été convoqués par la mairie et ils doivent rembourser les dégâts. Ça, mon père ça lui a mis un choc, la honte quoi! Il a honte de son fils. Il ne l'a pas frappé parce que ma mère l'a protégé, mais je crois qu'il l'aurait tué. Il a pas supporté son fils avec les flics, comme un... un bandit. Mais il nous inquiète lui, j'veux dire qu'il s'habille bien pour quelqu'un qui bosse pas beaucoup... Je doute un peu. Qu'est-ce que tu veux ? Il est dans la même chambre que mon autre frère, alors il voit... Et puis on fouine quand même dans ses affaires et tout. Son compte en banque est régulier donc... Enfin, il est vide quoi. A moins que, comme il est intelligent, il met ça directement dans la sape, je sais pas. On fouine mais sans être trop curieux, on regarde, quand ça sent trop la cigarette on se pose des questions - parce qu'il ne fume pas - alors on lui demande : 'T'as été où ? 
' ' Ça vous regarde pas!' Je crois pas qu'il vende. Mais j'ai peur qu'il soit complice d'eux, qu'il garde des trucs pour eux. La dernière fois, il avait plein de thunes sur lui. Je lui ai demandé d'où ça venait. Il m'a dit que c'était machin, là, qui est tombé et qui lui a demandé de garder cette thune. Je lui ai dit: 'T'as pas peur de mettre ça sur ton compte?'. ' Mais non, je le mets pas sur mon compte, je le laisse là '. Je lui dis : ' Mais imagine un jour qu'il y ait une inspection qui vient à la maison et qui trouve toute cette thune '. ' Mais non, mais non, je le garde momentanément après je lui rends'.

Mon père en a eu marre de lui, c'est pour ça qu'il a décroché. Nous, il nous a jamais vus comme ça. Nous, la sape, c'est quand on a commencé à travailler les étés, à avoir un peu d'argent. Mais un mec qui refuse d'aller en cours, qui préfère gagner de l'argent, qui est bien habillé... Parce que l'argent bon... On en a à la maison, on a toujours été nourris, logés, mes parents ne nous demandent rien. L'argent qu'on gagne c'est pour nous. C'est vrai que t'as toujours besoin d'un petit minimum, $1500 \mathrm{~F}$ par mois. Mais 9000-10000 F c'est autre chose... Quand tu vois grand, c'est que t'es intéressé par autre chose. C'est son problème. Il aime bien tout ce qui est grand, les belles choses... Là, je lui ai demandé ce qu'il avait envie de faire, il m'a dit: 'Je pars en Algérie'. Il veut partir à la fin du mois. Je sais pas ce qu'il fera là-bas... Mais c'est pas évident pour lui, même devant nous je veux dire. On n'a rien de plus que lui, mais on a le niveau terminale. On ne joue pas les fiers, on ne le rejette pas. C'est pas notre truc, ça. Au contraire, on est toujours en train de lui demander : vas-y, viens avec nous, tu vois? On ne joue pas le degré de supériorité avec lui, mais ça n'empêche, il se sent mal, il est mal à l'aise, même avec nous...»

En France depuis 1952, le père de Naïma est à l'image des pères algériens décrits par Abdelmalek Sayad, qui nourrissent sans doute d'autant plus des rêves de promotion improbable qu'ils entretiennent un rapport contradictoire à leur émigration. Voulant que leurs enfants réussissent et par-là justifient leur émigration (et d'abord à leurs propres yeux), ils se désolent en mesurant qu'ils ne sont pas les enfants qu'ils auraient pu être si la famille était restée en Algérie. Il y a peut-être dans la «folie des grandeurs " du père de Naïma une sorte de préparation inconsciente à la déception engendrée par « les enfants illégitimes » que ses fils et filles, nés et socialisés en France, sont voués à être à ses yeux. Le père de Naïma voyait sa fille aînée médecin, son premier fils architecte, sa fille cadette avocate, misait tout sur l'école. Mais s'il enjoignait ses enfants d'être à la hauteur de ses ambitions, il semblait dans le même temps vouloir limiter leur éloignement. Il leur rappelait en permanence qu'ils n'étaient pas vraiment des Français, qu'ils se leurraient à le croire, qu'ils étaient des Algériens comme leurs parents. Ainsi manifestait-il l'ambivalence caractéristique des pères appartenant à un groupe méprisé qui à la fois veulent que leurs enfants ne soient pas comme eux, qu'ils les dépassent, et qu'ils restent près d'eux, conformes à leur position et à leurs dispositions ${ }^{21}$. Il rêvait (et donc sans doute ne croyait-il pas complètement que les choses se passeraient dans la réalité comme il les imaginait) que ses enfants retourneraient avec lui en Algérie, dans la vaste maison qu'il a fait construire. Il n'a pas supporté qu'ils refusent de le suivre. Ayant intériorisé les normes du pays où ils sont nés, que leur père lui-même valorisait, les enfants ne peuvent pas s'identifier à la patrie de leurs parents.

Ces attentes contradictoires ne peuvent engendrer qu'un rapport malheureux aux enfants, quoi qu'ils fassent. D'où le dépit de Naïma qui, d'une certaine manière, semble dire à son père, à travers l'entretien, que ses enfants ne sont pas aussi « indignes » qu'il semble le croire. L'un de ses frères est diplômé d'un BTS et travaille régulièrement, sa 
sœur a récemment obtenu son baccalauréat et va préparer un DUT, elle-même veut sortir de l'usine : « réussites » moins brillantes que celles escomptées, mais volonté de mobilité néanmoins réelle, produit des dispositions inculquées dans la famille. Cependant, le père semble ne pas y accorder de valeur, sans doute parce que la modestie des trajectoires scolaires de ses enfants atteste à ses yeux qu'ils sont voués à être comme les autres, à l'identique des jeunes de leur condition.

Le « poids» des attentes déçues

Naïma met en évidence les attentes considérables des pères immigrés algériens, dont la recherche de reconnaissance est le moteur décisif des réussites scolaires parfois exceptionnelles de certains de leurs enfants, mais dont le caractère souvent intenable est aussi à l'origine des "blessures narcissiques" de ceux d'entre eux qui, jugés négativement à l'école, le sont de fait aussi et plus particulièrement dans la famille ${ }^{22}$. Aînée, Naïma endure les contradictions et la souffrance engendrées par le décalage entre les ressources culturelles réellement en sa possession et les attentes considérables de son père. Mais avec elle, c'est aussi son plus jeune frère qui est sous le " poids » de la déception du père. Elle décrit très bien les mécanismes qui le conduisent à être « le pire » des enfants : affecté de troubles du langage (il a été scolarisé dans une école spécialisée), il a un statut particulier dans la famille où il est le fils fragile. Son père n'attend pas de lui qu'il réussisse à l'Ecole et le destine à occuper un métier manuel. Autant dire qu'il le déclasse par rapport à ses autres enfants. Naïma observe qu'« il n'est pas à l'aise » parmi ses frères et sœurs, qu'il se sent inférieur à eux qui sont allés jusqu'au baccalauréat. Ayant interrompu sa scolarité avant le CAP, il semble se sentir « bête », moins "doué » qu'eux. D'ailleurs, contrairement au souhait du père, il n'étudie pas la mécanique : préserver son estime de soi, c'est pour lui refuser le destin par défaut, dévalué, auquel son père l'assigne. Fragile psychologiquement, démuni scolairement, se sentant marginalisé au sein de sa famille, il est prédisposé à la délinquance. Reconnu coupable d'un acte de vandalisme, il semble exprimer « la rage » propre aux jeunes sans avenir, qui cumulent tous les handicaps. Naïma redoute qu'il s'enfonce dans le trafic de drogue: outre les soutiens et les complicités qui le rehaussent (le réconfort de la bande), il semble lui procurer l'argent dont il a besoin. Sa sœur s'inquiète pour lui car "il aime bien tout ce qui est grand, les belles choses", le trafic étant la possibilité d'opposer au principe de domination fondé sur la possession de capital scolaire celui fondé sur la détention d'un capital économique. «Détenteur de valeurs de virilité périmées» - par la disparition des emplois ouvriers et la prolongation des scolarités - ayant intériorisé les normes de consommation dominantes, il est comme tant d'autres fils d'ouvriers démunis de capital scolaire voué à faire de la possession d'argent un substitut à la force physique dans la construction de son image de soi ${ }^{23}$.

Livré à lui-même par le retrait de son père, il l'est aussi par le fait que Naïma ne peut plus l'aider. Elle-même cherche à «se tirer d'affaire » (elle consacre son temps hors travail à trouver une formation qui la délivrera de son emploi d'OS). Elle se sent surtout discréditée aux yeux de son frère : l'échec dans ses études et son sentiment d'avoir «failli» lui interdisent d'exercer un rôle protecteur d'aînée. Privé d'avenir et de repères en France, son jeune frère pense partir en Algérie rejoindre son père, manifestant ainsi une recherche de reconnaissance paternelle autant que sa peur de «mal finir ». 
Menée à partir d'entretiens avec des parents algériens et marocains habitant dans un grand ensemble de la banlieue parisienne, cette enquête fait apparaître les processus multiples de fragilisation des habitants des $"$ cités $»^{24}$. La dégradation des conditions d'existence, provoquée par la réduction et la précarisation des emplois pas ou peu qualifiés et la disqualification des jeunes sans diplôme sur le marché du travail, explique le désarroi de ces parents. Leurs fils sont parmi les premiers affectés par le chômage de masse, l'élévation de la barrière à l'entrée du marché du travail, qui a résulté de la prolongation des scolarités, et subissent la discrimination à l'embauche, qui renforce leur relégation. Dans cette conjoncture, les fils d'immigrés non-diplômés sont comme voués à la délinquance : elle s'impose en effet à eux comme une ressource compensatoire, à travers «l'argent facile » et les profits symboliques immédiats qu'il procure, ne serait-ce qu'en termes d'apparence vestimentaire. Ainsi, contre l'approche ethno-culturaliste dominante qui tend à imputer les causes des désordres des cités aux propriétés culturelles des familles maghrébines, cet article rappelle leurs déterminants sociaux et économiques et fait apparaître que ces familles en sont les premières victimes.

La cité constitue pour elles une sorte de piège. Conséquence de la dévalorisation de l'habitat social et des logiques discriminantes qui vouent les familles immigrées aux secteurs dégradés des grands ensembles, le rassemblement de familles démunies dans les mêmes immeubles redouble le risque de voir les fils " mal tourner » ou " prendre la mauvaise pente ». La cité apparaît comme un facteur supplémentaire de fragilisation de familles ne détenant pas les ressources culturelles et économiques nécessaires pour préserver leurs enfants des sollicitations de la rue. De plus, les familles immigrées ne peuvent pas échapper individuellement à l'espèce d'opprobre collective qui entoure désormais le quartier. La délinquance ne menace pas seulement les familles démunies ou celles dont les fils sont désargentés et sans autre affectation que la rue. Elle renforce l'image que les plus dominants se font du groupe auquel elles appartiennent. Si l'accession au quartier avait pu concrétiser l'espoir d'une existence " normale ", égale à celle des ouvriers français, l'habiter représente aujourd'hui comme un retour en arrière dans l'infériorité. Ainsi la cité contribue-t-elle à atteindre les familles algériennes et marocaines dans leur honneur social et national.

La stigmatisation des parents algériens et marocains, perçus comme responsables des dégradations des jeunes, est un puissant facteur de division qui les empêche sans doute de s'organiser collectivement contre les causes sociales des désordres qu'ils subissent. L'enquête a mis en évidence que les pères et en particulier les pères algériens réagissent brutalement au sentiment de déshonneur et au risque d'une contamination par l'échec. Plus que les mères, ils sont en effet conduits à condamner les jeunes délinquants qui incarnent en quelque sorte la ruine des espoirs de promotion qu'ils avaient placés dans leur émigration. En s'éloignant physiquement ou symboliquement de leurs enfants devenus délinquants, certains d'entre eux manifestent avec force la désolation collective des pères. La mise en question de l'insertion professionnelle des jeunes à la sortie de l'école accroît la distance entre les pères algériens et leurs enfants. Séparés par les lieux de naissance et de socialisation, ils le sont aussi par la menace d'une retombée dans l'insécurité et l'indignitée ${ }^{25}$. Les mères tentent de préserver les liens familiaux. Elles s'opposent à la désaffiliation des fils «fautifs », en refusant de les exclure du domicile familial ou en ne «laissant pas tomber » ceux qui sont incarcérés. Manifestation de leur "habitus de mère", cette solidarité témoigne aussi de leur intériorisation de nouvelles «normes d'insertion » qui obligent les familles à assister 
plus longuement les jeunes scolarisés ou en attente d'un emploi. Partagées entre la condamnation morale publique et la compréhension privée des jeunes menacés dans leur avenir, les mères assurent ainsi tout un travail invisible sans lequel la solidarité entre les générations ne pourrait se maintenir ${ }^{26}$.

\section{NOTES}

1. . Cf. Michelle Tribalat, Dreux, voyage au cœur du malaise français, Paris, La Découverte et Syros, 1999.

2. . Cf. Pascal Duret, Anthropologie de la fraternité dans les cités, Paris, PUF, 1996.

3. Sur les scolarités des enfants d'immigrés algériens et marocains, voir notamment Malika Gouirir, Ouled el kharij, Les enfants de l'étranger. Socialisation et trajectoires familiales d'enfants d'ouvriers marocains immigrés en France, Paris X-Nanterre, 1997 ; Smaïn Laacher, «L'école et ses miracles. Note sur les déterminants sociaux des trajectoires scolaires des enfants des familles immigrées ", Politix, n¹2, 1990 ; Zaïhia Zéroulou, "La réussite scolaire des enfants d'immigrés », Revue française de sociologie, XXIX-3, juillet-septembre 1988.

4. . Entretiens réalisés dans le cadre de ma thèse qui porte plus généralement sur les transformations des conditions d'existence des classes populaires et leurs effets sur l'intégration des populations immigrées algériennes et marocaines, cf. Olivier Masclet, Rénovation urbaine et immigration : une intégration sous contrôle. Enquête sociologique dans une ville de la banlieue parisienne, thèse de l'EHESS, janvier 2001 (sous la direction de Gérard Mauger).

5. . Dans son bilan des recherches sur le contrôle parental, Laurent Mucchielli montre que ce contrôle est tributaire de l'environnement socio-économique. Voir L. Mucchielli, « Le contrôle parental du risque de délinquance : un bilan des recherches », Les Cahiers de la sécurité intérieure, Paris, $n^{\circ} 42,2000$. Nous insistons dans cet article sur la situation dominée des fils sur le marché du travail comme facteur de dégradation des rapports dans la famille et dans le quartier.

6. . Cf. Michel Pialoux, "Jeunes sans avenir et travail intérimaire », Actes de la recherche en sciences sociales, $n^{\circ} 26-27,1979$.

7. . Sur le rapport à l'avenir des jeunes des classes populaires et les conduites juvéniles qui en dépendent, voir Gérard Mauger, Claude Fossé-Poliak, « Les loubards », Actes de la recherche en sciences sociales, $n^{\circ} 50,1983$.

8. . Et à s'en protéger d'autant plus que le rassemblement dans les mêmes bâtiments de familles détenant peu de ressources a pour effet de redoubler la dépossession et de tirer vers le bas l'ensemble des habitants.

9. . Cf. Isaac Joseph, «Urbanité et ethnicité », Terrain, n³, 1984, p. 20-31.

10. . Cf. Abdelmalek Sayad, (avec Eliane Dupuy), Un Nanterre algérien, terre de bidonvilles, Paris, Editions Autrement, n85, avril 1995.

11. 1. Les mères et les sœurs « ne laissent pas tomber » leurs fils ou frères incarcérés, envoient des mandats, se rendent aux parloirs quand les pères y vont rarement, manifestent beaucoup moins de distance ou d'agressivité envers les surveillants de 
prison qui prennent leurs permis de visite ou leurs sacs de linge. En général, les pères et les fils s'évitent mutuellement. La honte des pères est si forte et si palpable qu'ils agressent leurs fils de récriminations amères et vaines. On pense à un détenu rencontré par Marie-Hélène Lechien qui explique avoir « interdit » à son père de venir, parce qu'il lui assène des « leçons de morale » éprouvantes, « lui prend la tête » et le laisse « sur les nerfs » lorsqu'il remonte en cellule. Il ne supporte plus ces reproches du père blessé qui non seulement défend son propre honneur, mais surtout continue à s'aveugler, à espérer que dès sa libération, le fils reprendra « le droit chemin ». Ce déni de la réalité est un rappel violent de l'impuissance des fils, qui savent leurs chances de « réinsertion » limitées mais comprennent la honte des pères. D'où l'évitement, l'évitement des blessures affectives et sociales qu'ils s'infligent mutuellement. Voir Marc Bessin et Marie-Hélène Lechien, « Soignants et malades incarcérés, Conditions, pratiques et usages des soins en prison ", GIP, novembre 2000.

12. . Sur les effets de l'allongement de la scolarité dans les milieux populaires, voir Jean-Pierre Terrail, Destins ouvriers, la fin d'une classe ?, Paris, PUF, 1990 ; Claude Grignon, « De l'école du peuple au lycée de masse », Critiques sociales, n³-4, nov. 1992 ; Gérard Mauger, « La reproduction des milieux populaires « en crise » ", Ville-EcoleIntégration, $\mathrm{n}^{\circ} 113,1998$; Stéphane Beaud et Michel Pialoux, Retour sur la condition ouvrière. Enquête aux usines Peugeot de Sochaux-Montbéliard, Paris, Fayard, 1999. 13. . Nous nous appuyons ici sur les observations d'un agent de la mission locale qui constate que « les patrons refusent d'embaucher des jeunes sans un minimum de bagage scolaire parce qu'ils les jugent trop indociles ». A ses yeux, ces jeunes sont « les moins insérables » : quand ils ne refusent pas les stages qui leur sont proposés, ils « font du bordel » et ne sont « jamais à l'heure».

14. . Cf. Stéphane Beaud et Michel Pialoux, Retour sur la condition ouvrière. Enquête aux usines Peugeot de Sochaux-Montbéliard, Paris, Fayard, 1999.

15. . Cf. François Dubet, « Comment devient-on ouvrier? », Autrement, « Ouvriers, ouvrières ", n¹26, janvier 1992.

16. . Numa Murard rappelle que l'éducation des adolescents s'effectue aussi « par la peur ». Les parents ouvriers sont aujourd'hui privés de cette ressource pour obliger leur fils à se plier aux normes, voir Numa Murard, « Autorité et amour : éducation des enfants ou mise en condition? », Mouvements, $n^{\circ} 8$, mars-avril 2000.

17. . Cf. Pierre Bourdieu, Méditations pascaliennes, Paris, Seuil, 1997, p. 275.

18. . Cf. Abdelmalek Sayad, « Les enfants illégitimes », L'immigration ou les paradoxes de l'altérité, Bruxelles, De Boeck, 1991, p. 185-258.

19. . Si cette famille n'est composée « que » de quatre enfants, ce n'est cependant pas en raison du projet de mobilité sociale des parents - projet qui a pour corollaire la réduction du nombre d'enfants - mais parce que la mère n'a pas pu avoir d'enfants rapidement après son mariage.

20. . Cf. Pierre Bourdieu, « Les contradictions de l'héritage », in Pierre Bourdieu (dir.), La Misère du monde, Paris, Seuil, 1993, p. 711-718.

21. . Cf. Pierre Bourdieu, « Les contradictions de l'héritage », op.cit.

22. . Ainsi peut-on expliquer pourquoi les enfants d'Algériens sont ceux qui à la fois réussissent le mieux et échouent le plus parmi les enfants d'immigrés, voir l'étude de Jean-Paul Caille et Louis André Vallet, « Les élèves étrangers ou issus de l'immigration dans l'école et le collège français ", Les Dossiers d'éducation et formations, $n^{\circ} 67$, Education nationale, avril 1996. 
23. . Voir Gérard Mauger, « Bandes et valeurs de virilité », Regards sur l'actualité, juillet-août 1998.

24. . Fragilisation inhérente à l'effritement de la société salariale, cf. Robert Castel, Les métamorphoses de la question sociale. Chronique du salariat, Paris, Fayard, 1995.

25. . Nous reprenons ici les analyses de Gérard Mauger sur les relations entre parents et enfants et les formes d'héritage dans les familles populaires. Cf. Gérard Mauger, « Les héritages du pauvre. Conflit œdipien et héritage social », Annales de la recherche urbaine, n41, 1989, p. 112-117.

26. . Voir Gérard Mauger, « Les héritages du pauvre », ibid.

\section{RÉSUMÉS}

A sociological inquiry into Maghrebi immigrant families' experiences in urban Paris distils interview data from Algerian and Moroccan parents. Findings reveal that most feel ashamed in their employment and housing situations. Mothers' and fathers' viewpoints are contrasted regarding their children and housing. Tenement living has weakened family morale and discipline. Causes and outcomes of young peoples' disillusionment with education are explored. Many families face a quandary of supporting young adults in a job sector of high unemployment. One father's disappointment in his children's accomplishment is illustrated in an extended interview excerpt. In contrast with the dominant ethno-culturist accounts, Maghrebi family disorders are attributed to social and economic factors that affect immigrants before others.

\section{INDEX}

Mots-clés : enfants, immigration, villes/ sociologie urbaine 